\title{
Probing adsorption on a nanoscale: field desorption microspectroscopy
}

\author{
Yuri Suchorski ${ }^{1}$ (D)
}

Received: 30 June 2016/Revised: 26 September 2016/Accepted: 30 September 2016/Published online: 7 October 2016

(C) The Author(s) 2016. This article is published with open access at Springerlink.com

\begin{abstract}
Combining an energy analyzer with a field ion microscope equipped with a probe-hole which corresponds to just few atomic surface sites, spatially resolved energy analysis of ions field desorbed from the adsorbent surface is possible on a nm-scale. The experimentally measured values of the kinetic energy of field ions can be related (by means of a thermionic cycle) to the physically meaningful binding energy of corresponding adsorbed species. The development of the technique into a full serviceable microspectroscopy on a nanoscale allowed recent detection of the weakly adsorbed $\mathrm{CO}$ species on $\mathrm{Pt}(111)$ which are largely analogous to those adsorbed at high pressures and provided first results for the binding energy of Li adatoms in a coadsorption system, namely Li-O-W(112) for various lithium and oxygen coverages. In the present contribution, an overview of the experimental possibilities of the technique is given and recent results are discussed.
\end{abstract}

Keywords Field desorption · Field ion microscopy · Field ion appearance energy spectroscopy · Coadsorption ·

Carbon monoxide $\cdot$ Lithium

\section{Introduction}

The term "microspectroscopy" is usually associated with a technique, where an X-ray or UV photon beam is focused into a fine spot and the energy of the locally emitted photoelectrons provides chemical information. By

Yuri Suchorski

yuri.suchorski@tuwien.ac.at

1 Institut für Materialchemie, Technische Universität Wien, Getreidemarkt 9, 1060 Vienna, Austria scanning the specimen with this spot, an image with chemical contrast can be achieved (Merkel et al. 2001). The high brightness of modern synchrotron radiation sources allows chemical surface imaging with resolutions in the $10 \mathrm{~nm}$ range (Ziethen et al. 2000). This is still far away from the ultimate chemical resolution, that is e.g. achieved with atom-probe (AP) techniques, where single atoms evaporated from the surface layer by high field (or laser) pulses are analysed (Suchorski and Drachsel 2011; Miller and Forbes 2014). However, the AP-approach aims at the 3D-analysis of the specimen (3D-tomography) rather than the adsorbed species on the surface and does not provide, at least in the usual commercial implementation, the binding energy of adsorbed molecules or atoms. ${ }^{1}$

However, in the last decades the demand for methods allowing the study of adsorption on the nm-scale scale has increased, due to fast widening of the assortment of available architectures of nanosized materials, such as nanoparticles, nanorods and nanosheets (Liu et al. 2013). The peculiarities of these novel nanoscale architectures, such as varying contribution of atoms located on confining surfaces of such nanosized material make such systems much more interesting, but additionally increase the difficulties of experimental studies. Even the mere imaging of the nm-scale pore arrangements appears difficult, without speaking about the in situ visualization of dynamic processes occurring in such systems. Although the local scanning techniques, such as STM and AFM allow real time observations of processes in an Angström range (Bliem et al. 2015), the studies of spatial correlation of events as well as the determination of the chemical identity of adsorbed species on an atomic scale or measuring of their binding energy still remain challenging.

\footnotetext{
${ }^{1}$ http://www.cameca.com/instruments-for-research/atom-probe.aspx.
} 
In turn, the field ion microscope (FIM), where the imaging ions are radially emitted from the nanotip surface creating an atomically resolved image, allows, due to the parallel imaging principle, the study of spatially correlated effects, such as diffusion (Binh 1983). A combination of an FIM with a small probe-hole in the screen also allows the local sampling of the surface species (Suchorski 2015). The species, field desorbed as ions from the surface, pass the probe-hole, which is adjusted to a few chosen surface sites, and are collected after a time-of-flight or magnetic sector field selection (Fig. 1). From the energy analysis of the selected ions the binding energy of adsorbed species can be determined, as will be explained in detail below. By moving the specimen, the probe-hole "scans" the specimen surface, providing spatially resolved spectroscopic information. Together with the atomically resolved image, simultaneously recorded from the screen, this realizes a microspectroscopy on the nm-scale. Since in the case of the magnetic sector field selection, a constant electric field can be used for field desorption, a "gentle" collecting of adsorbed species without damaging the specimen surface is possible.
This concept was first realized experimentally for adsorbed alkali atoms using a lithium field desorption microscope (Li-FDM), where the Li-ions field desorbed from the surface image the latter with a nearly atomic resolution (Medvedev et al. 1994), combined with a retarding potential analyzer. In this way, the binding energy of Li-adatoms field-desorbed from individual surface sites on W(111) was determined (Suchorski et al. 1995; 1996). Since that time, different adsorption systems such as $\mathrm{CO} / \mathrm{Pt}, \mathrm{N}_{2} / \mathrm{W}, \mathrm{O}_{2} / \mathrm{Pt}$, etc. were studied (Suchorski 2015). In the present contribution a short overview of our recent studies, namely the results for the weakly bound mobile CO adsorption layers, similar to those that exist during the high pressure adsorption and an example of the coadsorption (lithium and oxygen), are discussed.

\section{Principles and instrumentation}

Although the possibility to visualize individual surface atoms is the most impressive capability of the FIM based techniques, not less important is the ability to analyse the

(a)

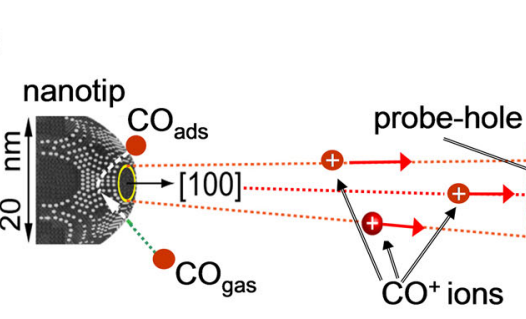

(b)

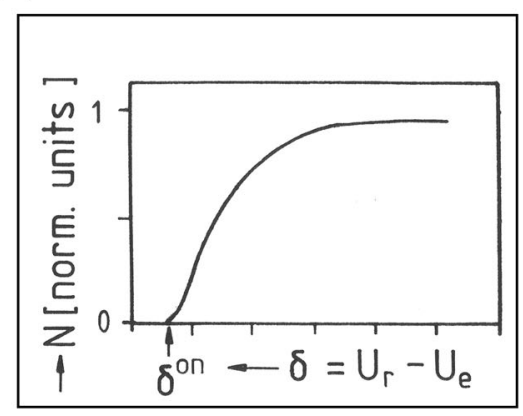

Fig. 1 Local mass-to-charge resolved retarding potential analysis of field desorbed species and determination of binding energies: a Scheme of the experimental set-up. The nanotip surface covered by adsorbed $\mathrm{CO}$ (as an example) is visualized in an FIM by $\mathrm{CO}^{+}$ions desorbed from the surface by high electric field. The $\mathrm{CO}^{+}$ions, which pass the probe hole in the middle of the screen and the magnetic sector field, are analysed in a retarding potential analyser. b A typical (c)

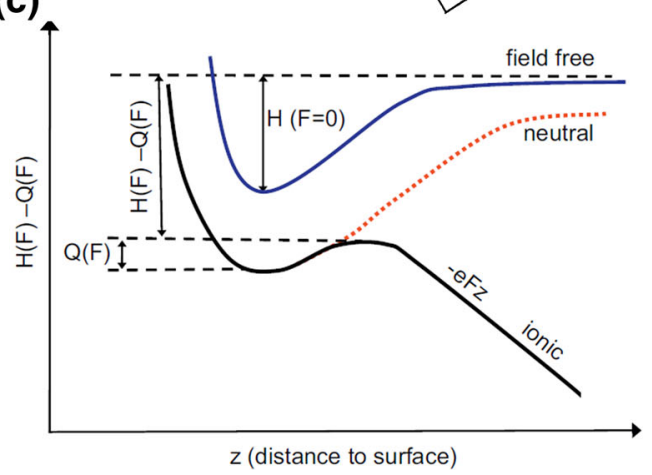

ion retardation curve, the potential difference $U_{r}-U_{e}$, at which the ions appear first, provides the onset voltage $\delta^{\text {on }}$. $\mathbf{c}$ Energy diagram illustrating the energy terms in Eq. (2). The neutral adsorption energy curve is bent by the externally applied field of $10-20 \mathrm{~V} / \mathrm{nm}$ so that adsorbed species can thermally escape (field-desorb) over the activation energy barrier 
ions of species emitted from the surface by applied electrostatic field. In the case of adsorbed species such process is called field desorption and, correspondingly, field evaporation, when the atoms of the specimen are emitted. For both processes, the applied field strains the binding between the surface and the atom or molecule going to be emitted, shifting it away from the equilibrium position, till the bond breaks. Therefore, the ions of atoms or molecules removed from the surface, are generated at a small distance $x$ (in Angström range) above the specimen surface and have thus an energy which is less than that of ions which would start directly at the surface. The latter would have an energy corresponding to the full accelerating potential of the specimen. The energy deficit of an amount $n e F x$, where $n e$ is the ionic charge and $F$ is the field strength near the tip surface, localizes thus precisely the field ionization event. The "bond breaking" distance and the corresponding energy deficit reflect the strength of the binding and thus the binding energy of the adsorbed species. A measurement of the energy deficits of field desorbed ions would then provide information on the site specific binding energy of adsorbed atoms or molecules. Energy deficits can be determined from the energy analysis of corresponding field ions: since after the field ionization event the total energy is conserved, the change of the potential energy at the instance of field ionization will contribute to the kinetic energy of the ion in the field-free region.

Of course, the complexity of the necessary instrumentation is much greater than that of conventional FIM, but the degree of the atomistic insight into the adsorption process pays off.

The technique consists of a probe-hole FIM setup combined with an instrument for mass-to-charge-resolved retarding potential analysis of field ions (Fig. 1a). In a probe hole experiment, the adsorbent-specimen (a nanotip, the apex of which is the studied surface) with an adsorbed layer (including multilayer adsorption) is exposed to a high electric field (V/nm range) which modifies the potential curve of adsorbed species (Fig. 1c), lowering the activation energy of desorption $Q(F)$. At sufficiently low $Q(F)$, a measurable flux of desorbed ions sets in and the ions (from few chosen surface sites) which pass the probe-hole are mass-to-charge selected in a magnetic sector field and then energy-analyzed with a five-electrode electrostatic retarding potential analyzer. By varying the threshold voltage $\delta=U_{\mathrm{r}}-U_{\mathrm{e}}$ (with $U_{\mathrm{r}}$ and $U_{\mathrm{e}}$ being the potentials of the retarder net and of the specimen emitting ions, respectively) the field ion retardation curves $N=N(\delta)$ can be obtained for any ions emitted from the selected surface sites on the tip surface (Fig. 1b). From these curves, the $\delta^{o n}$, onset voltage, can be determined, as a voltage at which ions just overcome the potential barrier and can be detected. From the onset voltage, the appearance energy of field ions, which is by definition the energy required to remove an electron from the neutral species to create a corresponding ground state ion, can be obtained as an empirical value

$A=n \phi_{\mathrm{ret}}-n e \delta^{o n}$

with $\phi_{\text {ret }}$ being the work function of the retarder (Goldenfeld et al. 1974).

The relation between the appearance energy of field ions and site specific binding energy of adsorbed species was derived theoretically by Forbes from the thermionic cycle long before the experimental realization (Forbes 1976). It provides the relationship between the appearance energy of field-desorbed ions, $A$, their ionization energy, $I$, the field dependent binding energy, $H(F)$ and the (also field-dependent) activation energy of field desorption, $Q(F)$ :

$A=I+H(F)-Q(F)$

The meaning of the terms $H(F)$ and $Q(F)$ is illustrated in Fig. 1c, note that $H(F)$ is the difference between the minimum of the total energy of the adsorbed atom or molecule in its ground state and the energy of the desorbed free molecule (in field-free space).

For many years, the application of Eqs. (1) and (2) for deriving the binding energies was hindered by problems of in situ determining the absolute work function of the retarder $\phi_{\text {ret }}$ and the onset voltage $\delta^{o n}$ simultaneously. While no problems appear with the onset voltage, the in situ work function measurements are complicated. A break-through was achieved in 1993 (Schmidt et al. 1993), by experimentally proving that at transition from localized to non-localized ionization of noble gases, the value of $A$ approaches exactly the ionization energy $I$, in full agreement with theoretical predictions (Forbes 1976). This made an in situ calibration of the work function of the retarder possible by substituting $A$ in Eq. (1) with the value $I$ for a noble probe-gas ionized at elevated temperature (Schmidt et al. 1994).

In the following application examples, $\mathrm{Ne}$ and $\mathrm{Ar}$, field ionized at $160 \mathrm{~K}$, i.e. at the temperature where the nonlocalized ionization takes place, were used for such calibration. Since at $160 \mathrm{~K}, A_{\mathrm{Ar}, 160 \mathrm{~K}}$ is equal to $I_{\mathrm{Ar}}$, the value of $\phi_{\text {ret }}$ could be obtainted in situ from Eq. (1). Once $\phi_{\text {ret }}$ is known, an absolute determination of the $A$ values for any other field-ionized species can be performed (in the same apparatus), including field-desorbed entities. Details of the experimental set-up including the tip assembly, adapted for liquid nitrogen cooling as well as for direct current heating (operating temperature between 79 and $600 \mathrm{~K}$ ), and the gas-supply systems were described elsewhere (Schmidt et al. 1994). The calibration of the applied electric field is based on the standard values of the evaporation field for corresponding metals (Müller and Tsong 1974). 
The development of the technique into a full serviceable micro-spectroscopy (where the probe hole can scan the surface during the simultaneous FIM imaging) on a nanoscale allowed recent detection of the weakly adsorbed $\mathrm{CO}$ species on $\mathrm{Pt}(111)$, which are largely analogous to those adsorbed at high pressures, and provided first measurements of the binding energy of particular adatoms in a coadsorption system, namely Li-O-W(112) for various lithium and oxygen coverages. These two application examples are reviewed below in detail.

\section{Application examples}

\subsection{Weakly adsorbed CO species on $\operatorname{Pt}(111)$}

The role of the weakly adsorbed $\mathrm{CO}$ species on Pt-catalyst surface, which are assumed to exist at elevated pressures, is often and controversially discussed in the literature (Freund et al. 2011; Farias et al. 2015). The experimental results are rather scarce due to the difficulties in detecting such layers in UHV. High electric field might stabilize such weakly bound layers facilitating the experimental verification. Figure 2 shows typical retardation curves for $\mathrm{CO}^{+}$and $\mathrm{Ar}^{+}$ ions emitted from the same few surface sites on the apex facet of a [111]-oriented $\mathrm{Pt}$ tip in the applied field range of 15-19 V/nm. The $\mathrm{Ar}^{+}$retardation curve shown on the right was used for the in situ determination of the work function of the retarder, as described above.

Retardation curves for $\mathrm{CO}^{+}$ions shown in (Fig. 2a) exhibit two remarkable features: (i) the onsets are shifted well above the values corresponding to the ionization energy threshold of the free CO molecule (marked by the dashed line) (ii) at increasing field strength the onsets shift to the right, i.e. the appearance energy $A$ increases. These observations are in good agreement with earlier findings for other $\mathrm{CO} / \mathrm{metal}$ systems and are in accord with the field desorption model of $\mathrm{CO}^{+}$ion formation (Schmidt et al. 1995). From the known $A$ values, the binding energy of CO molecules adsorbed on a $\mathrm{Pt}(111)$ nanofacet as a function of applied electric field can be obtained using Eq. (2), provided, the activation energy of field desorption, $Q(F)$, is known or can be neglected. The value of $Q(F)$, which can be derived from ion rate measurements, is in fact the effective energy which may be affected by the specifics of the diffusion supply of $\mathrm{CO}$ to the desorption sites ( $\mathrm{CO}$ from the tip-shaft replenishes the $\mathrm{CO}$ desorbed from the tip apex). A detailed analysis of the role of the supply (Suchorski et al. 1995 ; 1996) shows, however, that the effective values are close to the real $Q(F)$ values, as defined by the scheme in (Fig. 1c). For the present case, the $Q(F)$ value, as estimated from the measured temperature dependence of the $\mathrm{CO}^{+}$ rate, is lower than $0.1 \mathrm{eV}$, so that the $H(F)-Q(F)$ values
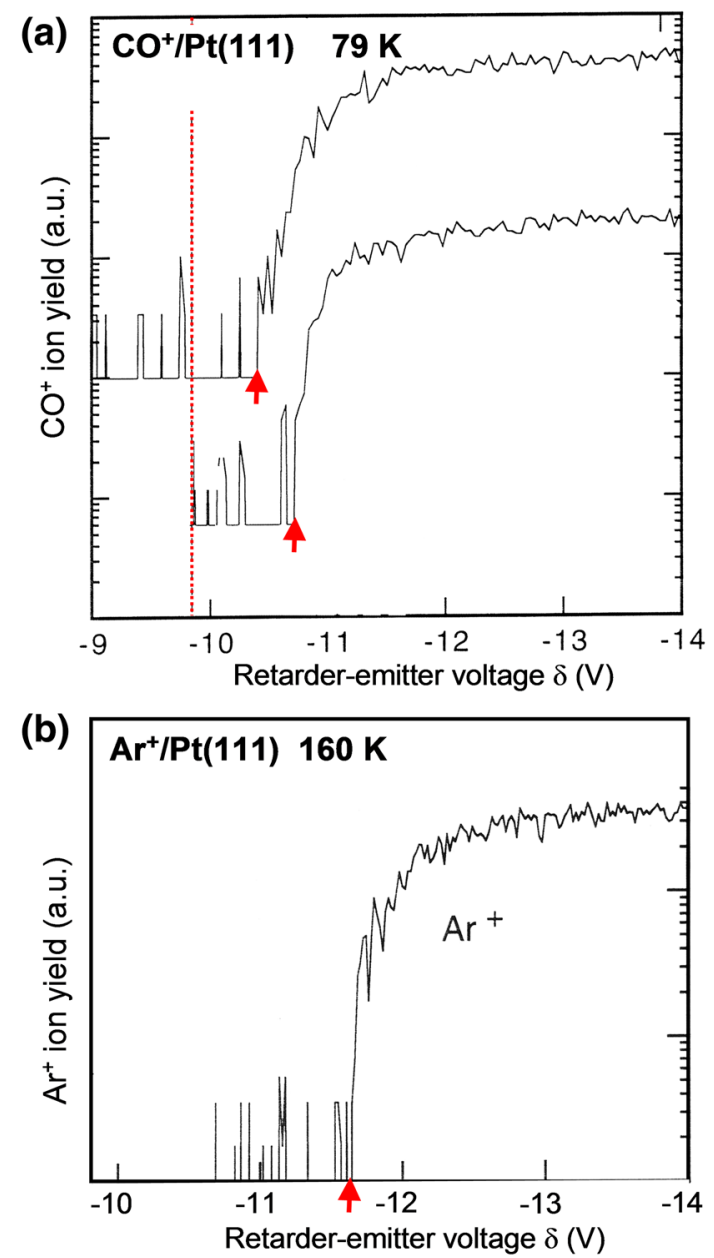

Fig. 2 Weakly bound CO species on a Pt(111) nanofacet: a Retardation curves for $\mathrm{CO}^{+}$ions emitted from $\mathrm{Pt}(111)$ at $15.4 \mathrm{~V} / \mathrm{nm}$ (upper curve) and at $18.9 \mathrm{~V} / \mathrm{nm}$ (lower curve) at $79 \mathrm{~K}$. The dotted line indicates the potential onset position for $A_{\mathrm{CO}}=I_{\mathrm{CO}}$. b The retardation curve for $\mathrm{Ar}^{+}$ions emitted from the same surfaces sites at $160 \mathrm{~K}$, which was used for calibration of $\phi_{\text {ret }}$ : the onset value read from the abscissa allows an in situ determination of $\phi_{\text {ret }}$ in Eq. (1)

can be interpreted as the binding energy $H(F)$ of $\mathrm{CO}$ molecules.

Figure 3 displays the values obtained for the binding energy of $\mathrm{CO}$ as a function of applied electric field. The $H(F)$ values range between 0.7 and $0.9 \mathrm{eV}$ for field strengths of 15-19 V/nm. The enhancement of the binding energy by the applied field can be understood on the basis of DFT calculations performed using the nonlocal BackePerdev exchange-correlation potential: the applied field stabilizes weakly bound $\mathrm{CO}$ molecules via additional fieldinduced charge transfer, enforcing the $\mathrm{Me}-\mathrm{C}-\mathrm{O}$ orientation (Schmidt et al. 1995). Because the field increases the binding energy, under field-free conditions the detected $\mathrm{CO}$ species would certainly exhibit a binding energy of $<0.7 \mathrm{eV}$. Comparison with adsorption calorimetry (Yeo et al. 1997), adsorption isotherms and thermal 

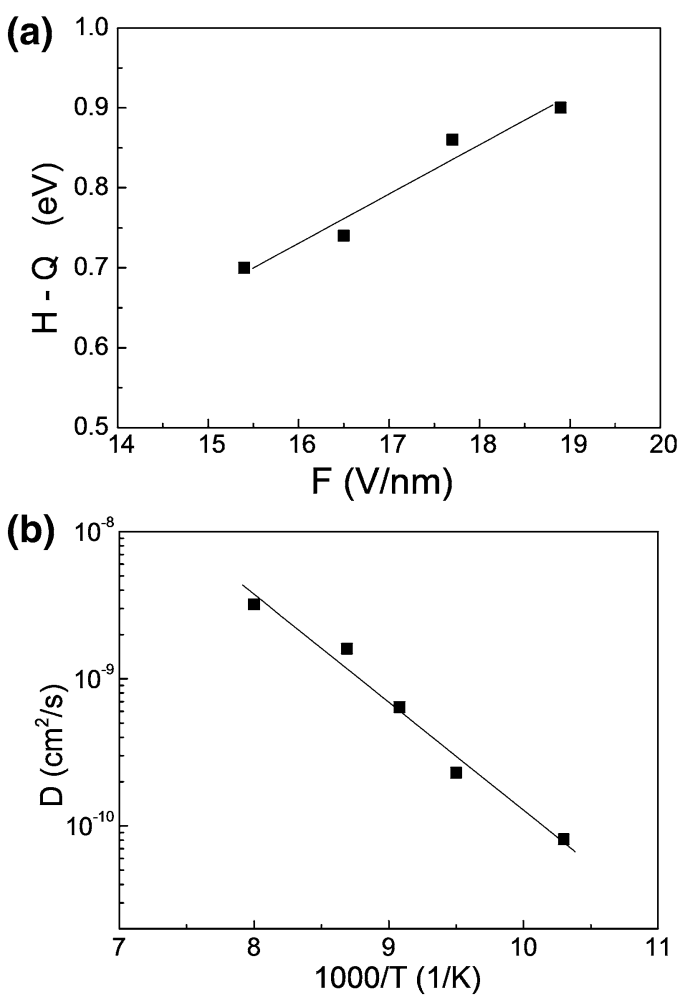

Fig. 3 a Field-dependent energy term $H(F)-Q(F)$ for neutral CO molecules on $\mathrm{Pt}(111)$ as a function of the externally applied field strength $F$. b Diffusion coefficient D for highly mobile CO species, being the origin of $\mathrm{CO}^{+}$ions emitted from a $\mathrm{Pt}(111)$ nanofacet, as a function of the inverse temperature (Arrhenius plot)

desorption results (Ertl et al. 1977), reporting a field-free binding energy of $\sim 0.65 \mathrm{eV}$ for $\Theta=0.7 \mathrm{ML}$, reveals that $\mathrm{CO}^{+}$detected in our experiments must originate from weakly bound species (at $\Theta>0.7 \mathrm{ML}$ ). For strongly bound $\mathrm{CO}$ species (at $\Theta<0.5 \mathrm{ML}$ ) the field-free binding energy is significantly higher $(>1 \mathrm{eV})$ (Yeo et al. 1997; Ertl et al. 1977), and we can thus exclude such adsorption states as origin of $\mathrm{CO}^{+}$field ions. Weakly bound $\mathrm{CO}$ species at high coverages were already reported for other platinum-metal surfaces: e.g. a binding energy of $0.34 \mathrm{eV}$ for $\Theta>0.8$ was derived in adsorption rate measurements for low temperature $\mathrm{CO}$ adsorption on $\mathrm{Rh}(100)$ (Medvedev et al. 1997), and a corresponding TPD peak at $100 \mathrm{~K}$ was observed for the same system (Peebles et al. 1985).

An evaluation of the diffusivity of $\mathrm{CO}$ molecules just before their field desorption corroborates the suggestion of a highly mobile $\mathrm{CO}$ layer being the origin of the field desorbed $\mathrm{CO}^{+}$ions. By "opening" the retarder, all $\mathrm{CO}^{+}$ ions that have passed the magnetic sector field can be registered and fluctuations of the $\mathrm{CO}^{+}$ion rate can be monitored. Because the fluctuations of number of collected $\mathrm{CO}^{+}$ions are directly related to the surface density fluctuation of $\mathrm{CO}$ molecules on the probed surface sites, the diffusivity of the $\mathrm{CO}$ species can be evaluated using the density fluctuation method as developed by Smoluchowski (1914). The physical basis for such an approach is Onsager's hypothesis, which states that the microscopic density fluctuation build-up and decay in accord with macroscopic diffusion laws (Onsager 1931). The FIM based techniques are naturally suitable for such approach, since they combine both, the high spatial resolution and the necessary coverage-sensitivity, and allow thus the monitoring of microscopic concentration inhomogeneities in a macroscopically homogeneous layer of adsorbed species [for a survey see e.g. Beben and Suchorski (2003)].

From the registered $\mathrm{CO}^{+}$ion rate fluctuations an autocorrelation function can be calculated $A_{\mathrm{N}}(t)=$ $\left\langle\delta N\left(t+t^{\prime}\right) \delta N\left(t^{\prime}\right)\right\rangle_{t^{\prime}}$ where $\delta N(t)$ is the fluctuation of $N$ at time $t$ and \langle\rangle$_{t^{\prime}}$ denotes the average over $t^{\prime}$. From the decay of the autocorrelation function the diffusion coefficient can be directly obtained, following the procedure described in our previous study (Suchorski et al. 1996). Figure $3 b$ shows the results of such an autocorrelation analysis of the rate fluctuations of $\mathrm{CO}^{+}$field ions emitted from a $\mathrm{Pt}(111)$ nanofacet as an Arrhenius plot. It is, however, difficult to compare the low activation energy of $0.11 \mathrm{eV}$, obtained for the present experiments for a stepped Pt-tip surface and an applied field of $15 \mathrm{~V} / \mathrm{nm}$, with known field-free data for single crystals [e.g. $0.13 \mathrm{eV}$ for diffusion over terraces and $0.31 \mathrm{eV}$ across steps (Ma et al. 1998)]. Nevertheless, despite the rather qualitative character of the obtained diffusivity and activation energy, the present diffusion experiments provide evidence for a highly mobile $\mathrm{CO}$ species being the origin of $\mathrm{CO}^{+}$ions detected by field ion appearance energy measurements.

In summary, the field-stabilization leads to an increased population of highly mobile $\mathrm{CO}$ molecules on the Pt tip surface, and thus to the presence of weakly bound $\mathrm{CO}$ molecules even at elevated temperatures. In the case of a catalytic reaction (e.g. $\mathrm{CO}$ oxidation), this affects the supply of $\mathrm{CO}$ into the reaction zone and modifies the dynamics of the reaction itself.

\subsection{Extracting Li-adatoms from the $\mathrm{Li}-\mathrm{O}$ coadsorption layers}

Alkali-oxygen coadsorption systems on metal surfaces are interesting both from the fundamental and practical point of view: such effects as alkali metal assisted oxidation of metal surfaces (Driver et al. 1997), or alkali promotion of the ammonia synthesis (Ertl 2003), Fischer-Tropsch reactions (Kiskinowa 1992), oxidation reactions such as ethanol- or CO-oxidation (Avgouropoulos et al. 2006; Pavlenko et al. 2001) are still in the focus of experimental and theoretical studies. 
Despite of intensive efforts, the mechanism of catalytic promotion by alkali coadsorption is still not entirely understood and remains a challenging task, because of the diversity of interactions involved in the coadsorption process. Additional complexity in the alkali-oxygen-substrate interaction might arise from the locally modified nanosized surface regions such as steps, kinks, defects etc. Unfortunately, it is difficult to study energetics of such effects, since existing experimental methods, frequently based on thermodesorption, do not provide adsorption energy data with the lateral resolution on a nanoscale. Therefore, the alkali-oxygen coadsorption is a good touch-stone for the laterally resolved measurements based on the appearance energy of field desorbed ions. Since the field desorption of $\mathrm{Li}$ from $\mathrm{W}$ occurs at RT already at applied fields of 5-8 V/ $\mathrm{nm}$ whereas more than $25 \mathrm{~V} / \mathrm{nm}$ are necessary to remove oxygen from the $\mathrm{W}$ surface, it is possible to extract $\mathrm{Li}$ from the coadsorbed $\mathrm{Li} / \mathrm{O}$-layer on tungsten.

A typical retardation curve for $\mathrm{Li}^{+}$ions emitted from few surface sites on the W(112) apex facet is shown in (Fig. 4a). The corresponding retardation curve for $\mathrm{Ne}^{+}$ ions emitted from the W(111) facet (Fig. 4b) was used for the in situ determination of the work function of the retarder, as described above. Retardation curves measured for $\mathrm{Li}^{+}$ions in the applied field range of 5-8 V/nm exhibit extremely steep onsets which are reflected in the extremely narrow energy distribution: the full width at half maximum (FWHM) for the $\mathrm{Li}^{+}$ions $(0.35 \mathrm{eV})$ is much smaller then for $\mathrm{Ne}^{+}$ions $(1.7 \mathrm{eV})$, due to an extremely high degree of spatial localization of the field desorption process. At increasing field strength the onset values $\delta^{\text {on }}$ shift to the right, the appearance energy $A_{\mathrm{Li}}$ increases. These observations are in good agreement with earlier findings for $\mathrm{Li}$ desorbed from a pure Li layer (Suchorski et al. 1995; 1996).

Like in the previous case of $\mathrm{CO}$, the binding energy of Li adatoms coadsorbed with oxygen can be obtained from the appearance energy $A_{\mathrm{Li}}$ values. However, in the present case of $\mathrm{Li}$, the activation energy of field desorption, $Q(F)$, is higher than that of $\mathrm{CO}$ and cannot be neglected. It can be, however, determined from the rate measurements.

The value of the appearance energy $A_{\mathrm{Li}}=7.29 \mathrm{eV}$ obtained from the $\delta^{\text {on }}$ in (Fig. 2a), ionization energy $I_{\mathrm{Li}}=5.39 \mathrm{eV}$ and activation energy $\mathrm{Q}=0.2 \mathrm{eV}$ provide, using the Eq. (2) the value of $2.1 \mathrm{eV}$ for binding energy of $\mathrm{Li}$ in a coadsorbed $\mathrm{Li} / \mathrm{O}$ layer consisting of $1 \mathrm{ML}$ of $\mathrm{Li}$ and $0.5 \mathrm{ML}$ of oxygen. The binding energy value appeared to be field independent, at least in the used range $8-13 \mathrm{~V} / \mathrm{nm}$, as is also expected from the physical picture of alkali adsorption (Suchorski et al. 1996). It was also possible to study a two-layer configuration: the corresponding binding energy of $\mathrm{Li}$ desorbed from the second adsorption layer decreased to $1.8 \mathrm{eV}$. The measured values are independent of the evaporation order: either the $\mathrm{Li}$ was evaporated on the oxygen-precovered surface or oxygen was dosed to $\mathrm{Li}$ evaporated on tungsten, also any lithium oxide ions were not detected in our experiments.

Comparison of present values of the binding energy of $\mathrm{Li}$ in $\mathrm{Li} / \mathrm{O}$-coadsorbed layers with the data obtained for the same $\mathrm{Li}$ and $\mathrm{O}$ coverage on the single W(112) crystal surface [using the adsorption-desorption equilibrium measurements (Suchorski and Hupalo 2011)], reveal a quantitative agreement of the data obtained locally for few atomic sites on a nm-sized W(112) facet and macroscopic single crystal surface, correspondingly.

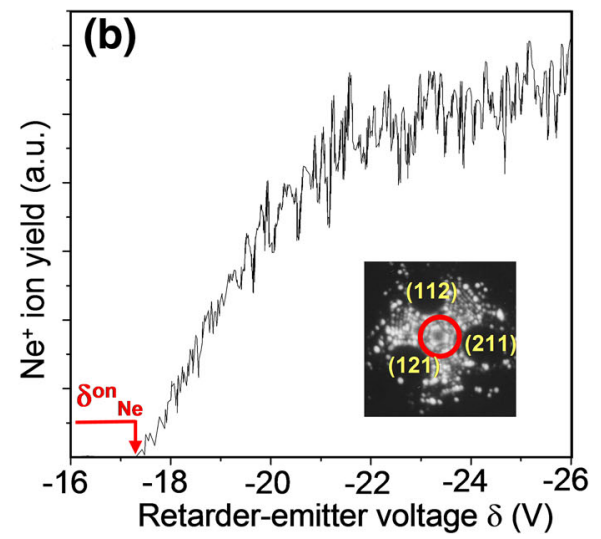

$335 \mathrm{~K}$ and $13 \mathrm{~V} / \mathrm{nm}$ ) and the position of the probe-hole, $\mathbf{b}$ the same, but for the $\mathrm{Ne}^{+}$ions emitted from the $\mathrm{W}(111)$ facet at $160 \mathrm{~K}$ and $35 \mathrm{~V} / \mathrm{nm}$. The retardation curve $\mathrm{N}(\delta)$ was used for the in situ $\Phi_{\text {ret }}$ calibration. The inset shows a $\mathrm{Ne}^{+}$FIM image (at $78 \mathrm{~K}$ and $35 \mathrm{~V} / \mathrm{nm}$ ) and the position of the probe-hole 


\section{Summary and outlook}

In summary, presented exemplary results, obtained by laterally resolved field ion appearance energy spectroscopy, demonstrate, that such kind of spectroscopy, combined with atomically resolving microscopy indeed provides interesting insights into the adsorption processes: the field-stabilization of adsorbed CO leads to an increased population of highly mobile $\mathrm{CO}$ molecules on the Pt tip surface, and thus to the presence of weakly bound $\mathrm{CO}$ molecules even at elevated temperatures. In the case of catalytic reactions, e.g. $\mathrm{CO}$ oxidation, this affects the supply of $\mathrm{CO}$ into the reaction opening a route to (partly) bridging the pressure gap. The above measurements can be carried out at system pressures up to $10^{-4}$ mbar. In addition, the field-induced attraction of polarized gas species [the so called "field-compression" effect (Rendulic and Leisch 1980)] enhances the local pressure at the tip surface up to 10 times (depending on gas polarizability), thus approaching the $10^{-2}$ mbar range (which is ca. $10^{4}$ times higher in pressure than conventional TPD analysis). This increases the local adsorbate coverage and significantly modifies reaction processes. For example, for $\mathrm{CO}$ oxidation on a Pt-tip (at $298 \mathrm{~K}$ and $\mathrm{p}_{\mathrm{O} 2}=4 \times 10^{-4} \mathrm{mbar}$ ) in the presence of an applied field of 10-15 V/nm the maximum reaction rate was observed for a $\mathrm{CO}$ pressure in the $10^{-5}$ mbar range. For field-free conditions a 6-times higher $\mathrm{CO}$ pressure was required to observe the same maximum on the same Pt-tip (Suchorski et al. 1998).

The apex of a nanotip, as used in present measurements mimics well a single catalytic nanoparticle, exhibiting the same main feature, namely crystallographically differently oriented nanofacets. In contrary to a particle, the surface of the nanotip can be prepared and characterized with atomic resolution (Suchorski and Drachsel 2007). Consequently, using a nanotip as a model catalyst might allow to overcome, at least partially, both the materials and pressure gap. It is thus interesting to compare the above results of $\mathrm{CO}$ adsorption on a $\mathrm{Pt}(111)$ nanofacet with corresponding high pressure (mbar-range) studies for a $\operatorname{Pt}(111)$ single crystal surface using vibrational sum frequency generation (SFG) (Rupprechter et al. 2003). The SFG data for Pt(111) identified a similar dense $\mathrm{CO}$ adlayer with $\sim 0.7 \mathrm{ML}$ coverage as was detected by high-field studies under vacuum conditions, whereas field ion spectroscopy additionally provided information on the binding energy and diffusivity of weakly bound highly mobile $\mathrm{CO}$ species, that may be the active species in $\mathrm{CO}$ oxidation reaction.

Novel nanosized materials, such as nanoparticles, nanosheets, and particularly nanorods are suitable specimens for such kind of microspectroscopy, where adsorbed species emitted from e.g. a single nanopore, both from a monolayer or multilayer, might be studied in this way. Moreover, species generated in situ during a catalytic reaction can be analyzed.

Acknowledgments This work was supported by the Austrian Science Fund (FWF) [SFB F45 FOXSI]. Open access funding provided by Austrian Science Fund (FWF).

Open Access This article is distributed under the terms of the Creative Commons Attribution 4.0 International License (http://crea tivecommons.org/licenses/by/4.0/), which permits unrestricted use, distribution, and reproduction in any medium, provided you give appropriate credit to the original author(s) and the source, provide a link to the Creative Commons license, and indicate if changes were made.

\section{References}

Avgouropoulos, G., Oikonomopoulos, E., Kanistras, D., Ioannides, T.: Complete oxidation of ethanol over alkali-promoted $\mathrm{Pt} / \mathrm{Al}_{2} \mathrm{O}_{3}$ catalysts. Appl. Catal. B Environ. 65, 62-69 (2006)

Beben, J., Suchorski, Y.: Surface diffusion by adsorbate density fluctuation measurements. Prog. Surf. Sci. 74, 3-24 (2003)

Binh, V.T.: Surface Mobilities on Solid Materials: Fundamental Concepts and Applications, vol. 86. Springer, Boston (1983)

Bliem, R., van der Hoeven, J., Zavodny, A., Gamba, O., Pavelec, J., de Jongh, P.E., Schmid, M., Diebold, U., Parkinson, S.G.: An atomic-scale view of $\mathrm{CO}$ and $\mathrm{H}_{2}$ oxidation on a $\mathrm{Pt} / \mathrm{Fe}_{3} \mathrm{O}_{4}$ model catalyst. Angew. Chem. Int. Ed. 54, 13999-14002 (2015)

Driver, S.M., Lüdecke, J., Dixon, R.J., Thompson, P.B.J., Scragg, G., Woodruff, D.P., Cowie, B.C.C.: Alkali-promoted oxidation of $\mathrm{Al}(111): \mathrm{Rb} / \mathrm{O}$ and $\mathrm{K} / \mathrm{O}$ coadsorption and the role of surface structure. Surf. Sci. 391, 300-314 (1997)

Ertl, G.: Ammonia synthesis-heterogeneous. In: Horvath, I.T. (ed.) Encyclopedia of Catalysis, vol. 1, pp. 329-352. Wiley, Hoboken (2003)

Ertl, G., Neumann, M., Streit, K.M.: Chemisorption of CO on the Pt(111) surface. Surf. Sci. 64, 393-410 (1977)

Farias, M.J.S., Camara, G.A., Feliu, J.M.: Understanding the CO preoxidation and the intrinsic catalytic activity of step sites in stepped pt surfaces in acidic medium. J. Phys. Chem. C 119(35), 20272-20282 (2015)

Forbes, R.G.: A generalised theory of standard field ion appearance energies. Surf. Sci. 61, 221-240 (1976)

Freund, H.-J., Meijer, G., Scheffler, M., Schlögl, R., Wolf, M.: CO oxidation as a prototypical reaction for heterogeneous processes. Angew. Chem. Int. Ed. 50(43), 10064-10094 (2011)

Goldenfeld, I.V., Korostyshevsky, I.Z., Michanchuk, B.G.: Analysis of field ion energies in a mass spectrometer. Int. J. Mass Spectrom. Ion Phys. 61, 211 (1974)

Kiskinowa, M.P.: Poisoning and promotion in catalysis based on surface science concepts and experiments, Studies in Surface Science and Catalysis, vol. 70. Elsevier, Amsterdam (1992)

Liu, Z., Fujita, N., Miyasaka, K., Han, L., Stevens, S.M., Suga, M., Asahina, S., Slater, B., Xiao, C., Sakamoto, Y., Anderson, M.W., Ryoo, R., Terasaki, O.: A review of fine structures of nanoporous materials as evidenced by microscopic methods. Microscopy 62, 109-146 (2013)

Ma, J., Xiao, X., DiNardo, N.J., Loy, M.M.T.: Diffusion of CO on Pt(111) studied by an optical diffraction method. Phys. Rev. 58, 4977-4983 (1998) 
Medvedev, V.K., Suchorski, Y., Block, J.H.: Lithium field desorption microscope: a new tool for surface investigations. Ultramicroscopy 53, 27-35 (1994)

Medvedev, V.K., Kulik, V.S., Chernyi, V.I., Suchorski, Y.: Weakly chemisorbed $\mathrm{CO}$ layer on $\mathrm{Rh}(100)$ as detected by reverse flash and field ion appearance energy measurements. Vacuum 48, 341-345 (1997)

Merkel, M., Escher, M., Settemeyer, J., Funnemann, D., Oelsner, A., Ziethen, C., Schmidt, O., Klais, M., Schönhense, G.: Microspectroscopy and spectromicroscopy with photoemission electron microscopy using a new kind of imaging energy filter. Surf. Sci. 480, 196-202 (2001)

Miller, M.K., Forbes, R.G.: Atom-Probe Tomography-The Local Electrode Atom Probe. Springer, Heidelberg (2014)

Müller, E.W., Tsong, T.T.: Field ion microscopy, field ionization and field evaporation. Prog. Surf. Sci. (1974). doi:10.1016/S00796816(74)80005-5

Onsager, L.: Reciprocal relations in irreversible processes. Phys. Rev. 38, 2265-2279 (1931)

Pavlenko, N., Kostrobij, P.P., Suchorski, Y., Imbihl, R.: Alkali metal effect on catalytic $\mathrm{CO}$ oxidation on a transition metal surface: a lattice-gas model. Surf. Sci. 489, 29-36 (2001)

Peebles, H.C., Beck, D.D., White, J.M., Campbell, C.T.: Structure of $\mathrm{Ag}$ on $\mathrm{Rh}$ and its effect on the adsorption of $\mathrm{D}_{2}$ and CO. Surf. Sci. 150, 120-142 (1985)

Rendulic, K.D., Leisch, M.: The gas supply function in field ion microscopy. Surf. Sci. 95, L271-L272 (1980)

Rupprechter, G., Unterhalt, H., Morkel, M., Galetto, P., Dellwig, G., Freund, H.-J.: Extending UHV studies to the mbar range: vibrational SFG spectroscopy of high pressure $\mathrm{CO}$ adsorption on $\operatorname{Pt}(111)$ and $\operatorname{Pd}(111)$. Vacuum 71, 83-87 (2003)

Schmidt, W.A., Ernst, N., Suchorski, Y.: Local electric fields at individual atomic surface sites: field ion appearance energy measurements. Appl. Surf. Sci. 67, 101-110 (1993)

Schmidt, W., Suchorski, Y., Block, J.: New aspects of field adsorption and accommodation in field ion imaging. Surf. Sci. 301, 52-60 (1994)

Schmidt, W.A., Suchorski, Y., Block, J.H., Kreuzer, H.J., Wang, R.L.C.: Field ion appearance energy spectroscopy of $\mathrm{CO}^{+}$ originated from $\mathrm{Rh}(111)$ and $\mathrm{Au}(111)$ surface step sites. Surf. Sci. 326, 243-251 (1995)
Smoluchowski, M.: Studien über Molekularstatistik von Emulsionen und deren Zusammenhang mit der Brown'schen Bewegung. Sitzungsberichte der Wiener Akademie 123, 2381-2405 (1914)

Suchorski, Y.: Field ion and field desorption microscopy: principles and applications. In: Kumar, C.S.S.R. (ed.) Surface Science Tools for Nanomaterials Characterization, pp. 2-43. Springer, Heidelberg (2015a)

Suchorski, Y.: Field ion and field desorption microscopy: surface chemistry applications. In: Reedijk, J. (ed.) Reference Module in Chemistry, Molecular Sciences and Chemical Engineering, pp. 1-18. Elsevier, Waltham (2015b)

Suchorski, Y., Drachsel, W.: Catalytic reactions on platinum nanofacets: bridging the size and complexity gap. Top. Catal. 46, 201-215 (2007)

Suchorski, Y., Drachsel, W.: Field ion microscopy (FIM) and atom probe (AP). In: Friedbacher, G., Bubert, H. (eds.) Surface and Thin Film Analysis, pp. 237-261. Wiley, Weinheim (2011)

Suchorski, Y., Hupalo, M.S.: Coadsorption of lithium and oxygen on $\mathrm{W}(112)$ : nanosized facets versus single crystals. Ultramicroscopy 111(6), 381-385 (2011)

Suchorski, Y., Medvedev, V., Block, J.: Absolute appearance energy of li ions field-desorbed from W(111). Phys. Rev. B 51(7), 4116-4734 (1995)

Suchorski, Y., Medvedev, V., Block, J., Wang, R., Kreuzer, H.: Field desorption of lithium. Phys. Rev. B 53, 4109-4116 (1996a)

Suchorski, Y., Beben, J., Medvedev, V.K., Block, J.H.: Study of CO surface diffusion on $\mathrm{CO} / \mathrm{W}(111)$ by analysis of $\mathrm{CO}^{+}$field ion rate fluctuations. Appl. Surf. Sci. 94-95, 207-211 (1996b)

Suchorski, Y., Imbihl, R., Medvedev, V.K.: Compatibility of field emitter studies of oscillating surface reactions with single crystal measurements: catalytic CO oxidation on Pt. Surf. Sci. 401(3), 392-399 (1998)

Yeo, Y.Y., Vattuone, L., King, D.A.: Calorimetric heats for CO and oxygen adsorption and for the catalytic $\mathrm{CO}$ oxidation reaction on Pt $\{111\}$. J. Chem. Phys. 106, 392 (1997)

Ziethen, C., Schmidt, O., Marx, G.K.L., Schönhense, G., Frömter, R., Gilles, J., Kirschner, J., Schneider, C.M., Gröning, O.: Orbital mapping of carbon thin films by XANES-spectromicroscopy. J. Electron Spectrosc. 107, 261-271 (2000) 